\title{
The small leucine-rich proteoglycan, biglycan, is highly expressed in adipose tissue of Psammomys obesus and is associated with obesity and type 2 diabetes
}

\author{
This article was published in the following Dove Press journal: \\ Biologics:Targets and Therapy \\ 30 March 2012 \\ Number of times this article has been viewed
}

\section{Kristy Bolton' \\ David Segal' \\ Ken Walder ${ }^{1,2}$}

'Metabolic Research Unit, School of Medicine, ${ }^{2}$ Institute for Technology, Research and Innovation, Deakin University, Waurn Ponds, Victoria, Australia
Correspondence: Kristy Bolton Metabolic Research Unit, School of Medicine, Deakin University, Pigdons Rd, Waurn Ponds, Victoria, Australia 3217

Tel +6I 352278425

Fax+6I 352278376

Email kabolton@deakin.edu.au
Abstract: We have previously demonstrated that the small leucine-rich proteoglycan decorin may play a role in adipose tissue homeostasis and the pathophysiology of obesity. Biglycan is highly similar in structure to decorin, therefore we hypothesized it would have a similar expression profile and role to decorin in adipose tissue. Real time polymerase chain reaction was used to measure biglycan mRNA levels in adipose tissue from normal glucose tolerant and impaired glucose tolerant and type 2 diabetic (T2D) Psammomys obesus. Biglycan mRNA was found to be highly expressed in adipose tissue, and gene expression was significantly higher in visceral compared to subcutaneous adipose tissue, with elevated levels in obese, T2D compared to lean normal glucose tolerant $P$. obesus $(P<0.04)$. Biglycan mRNA was predominantly expressed by stromal/vascular cells of fractionated adipose tissue $(P=0.023)$. Biglycan expression in adipose tissue, particularly in the obese state, was markedly upregulated. Collectively, our data suggest that the small leucine-rich proteoglycan family proteins biglycan and decorin may play a role in the development of obesity and T2D, possibly by facilitating expansion of adipose tissue mass.

Keywords: biglycan, small leucine-rich proteoglycan, Psammomys obesus, adipose tissue, obesity, type 2 diabetes

\section{Introduction}

We previously identified the small leucine-rich proteoglycan (SLRP) decorin as a secreted protein not previously associated with the development of obesity, type 2 diabetes (T2D), and the metabolic syndrome. ${ }^{1}$ Decorin was highly expressed in adipose tissue, with significantly higher gene expression in visceral compared to subcutaneous adipose tissue depots in both Psammomys obesus and human subjects $(P=0.002$ and $P=0.001$, respectively). ${ }^{1}$ Furthermore, decorin was shown to be expressed adjacent to blood vessels in the adipose tissue. ${ }^{1}$ These findings suggest that this SLRP may play a role in adipose tissue homeostasis and in the pathophysiology of obesity.

Biglycan is a SLRP closely related to decorin, with sequence and structure analysis showing the genes are 55\% homologous at the amino acid level and are most likely a product of gene duplication. ${ }^{2}$ Like decorin, biglycan binds transforming growth factor $\beta^{3}$ and interacts with collagen. ${ }^{4}$ The similarity in structure and overlapping expression profiles in skeletal muscle and connective tissues suggest that it is likely that these two proteins function redundantly and synergistically. ${ }^{5-7}$ The targeted disruption of decorin in mice results in mice with fragile skin, reduced tensile strength, and abnormal 
collagen morphology, ${ }^{8}$ whereas biglycan knockout mice have an osteoporosis-like phenotype. ${ }^{9}$ The biglycan knockout mice are born with no apparent defects however after 3 months they have a reduced growth rate and decreased bone mass suggesting biglycan is a postnatal regulator of skeletal mass. ${ }^{9}$ Previous studies investigating the biglycan, ${ }^{9}$ decorin, ${ }^{8}$ and biglycan/decorin ${ }^{10}$ deficient mouse models have suggested that these two genes may have redundant functions, and furthermore, may compensate for each other's functions when one of them is deficient. ${ }^{6,11}$ Mice deficient in decorin and biglycan exhibit a more severe phenotype than mice deficient in either gene alone. ${ }^{10}$ Biglycan and decorin therefore may partly rescue or compensate for each other's absence in the singly deficient mice. ${ }^{7,11-13}$

Given the expression and functional similarity between decorin and biglycan, the aim of this study was to characterize biglycan in relation to obesity and T2D in P. obesus.

\section{Materials and methods Experimental animals}

A colony of $P$. obesus were maintained at Deakin University as previously described. ${ }^{1}$ At 16 weeks of age, animals were classified into three groups according to their blood glucose and plasma insulin concentrations. ${ }^{1,14}$ The groups were as follows: (1) lean and normal glucose tolerant (NGT), (2) overweight and impaired glucose tolerant (IGT), and (3) obese, T2D. Phenotypic characteristics of each group are previously published. ${ }^{1}$ At 18 weeks of age, animals were killed by anesthetic overdose (pentobarbitone, $120 \mathrm{mg} / \mathrm{kg}$; Sigma, St Louis, MO) and tissues were rapidly excised, snap frozen in liquid nitrogen, and stored at $-80^{\circ} \mathrm{C}$ for subsequent RNA extraction. All experiments were conducted according to strict National Health and Medical Research Council guidelines and approved by the Deakin University Animal Welfare Committee.

\section{RNA extraction}

Total RNA was extracted from $P$. obesus ( $\mathrm{n}=5-6$ per group) tissues using TRIzol (Invitrogen, Carlsbad, CA) in conjunction with RNeasy columns (Qiagen, Hilden, Germany). The quality and concentration of RNA was determined using the RNA 6000 Nano Assay and an Agilent 2100 Bioanalyzer (Agilent Technologies, Santa Clara, CA). Total RNA was extracted in this study from the following tissues: testes; lung, small and large intestine; adrenal; spleen; kidney; stomach; heart; perirenal, mesenteric, epididymal, intramuscular, and subscapular adipose tissue; soleus, extensor digitorum longus, plantaris, white and red gastrocnemius; liver; brain stem, mid brain, hippocampus, cerebellum, cortex, and hypothalamus. With regards to adipose tissue depots, brown adipose tissue depots were excised and discarded in order to freeze only the white adipose tissue in each depot type. The intramuscular fat originates from a fat depot associated with the gastrocnemius muscle.

\section{Real time polymerase chain reaction}

First strand cDNA was generated from RNA using SuperScript First-Strand Synthesis System (Invitrogen) for real time polymerase chain reaction (RT-PCR). Biglycan gene expression levels were quantitated using SYBR Green PCR Master Mix (Applied Biosystems, Worthington, UK) with an ABI PRISM 7700 Sequencing Detector (Perkin Elmer, Waltham, MA). Arbitrary units (AU) were calculated using the delta cycle threshold method. Gene expression was normalized to the group of samples with the lowest mean cycle threshold. RT-PCR primers were as follows: P. obesus biglycan forward 5'-GACAACCGTATCCGCAAAGTG -3', reverse 5'-GAGCTTCAGGCCATCAAAGG -3'.

\section{Adipose tissue fractionation}

P. obesus $(\mathrm{n}=5)$ were sacrificed and the epididymal adipose tissue was removed and digested with type I colleganse digestion as previously described. ${ }^{1}$ Fractions were frozen in liquid nitrogen for subsequent RNA extraction and RT-PCR as described above.

\section{Statistical analysis}

Statistical analysis was performed using SPSS (v 14.0; SPSS Inc, Chicago, IL). Levene's test for homogeneity of variance was used to determine if variance between two groups was equal. As homogeneity was equal, a one-way analysis of variance with a post hoc least significant difference test was used. Associations between gene expression levels and phenotypic measures were determined using Pearson's correlation for normally distributed data or Spearman's correlation for data that were not normally distributed. To compare mean phenotypic values between groups, independent samples $t$-test was used. Differences and correlations were considered significant at $P<0.05$.

\section{Results}

\section{Biglycan is highly expressed in adipose tissue from P. obesus}

Biglycan gene expression was analyzed in a variety of tissues from $P$. obesus, an animal model of obesity and T2D. Similar to decorin, biglycan had markedly higher expression 
within adipose tissue depots compared with all other tissues examined (Figure 1). Biglycan was also expressed at lower levels in the brain, liver, red gastrocnemius, heart, stomach, kidney, spleen, adrenal, lung, and testes.

Due to the remarkable expression within adipose tissue depots, biglycan gene expression was next analyzed in visceral (mesenteric) and subcutaneous (subscapular) adipose tissue of P. obesus by RT-PCR. Biglycan expression was higher in visceral compared to subcutaneous adipose tissue overall ( $P=0.038$, gene expression (mean AU \pm standard error of mean): visceral $655 \pm 123$, subcutaneous $357 \pm 61$ ). In P. obesus with impaired glucose tolerance, biglycan gene expression was significantly higher in visceral compared with subcutaneous adipose tissue ( $P=0.049$, Figure 2A). Separately, within subcutaneous tissue, biglycan gene expression was significantly elevated in IGT and T2D P. obesus compared with healthy (NGT) littermates $(P=0.009$ and $P<0.001$, respectively) and significantly correlated with body weight $\left(\mathrm{r}^{2}=0.38, P=0.012\right)$, plasma glucose, and insulin concentration $\left(\mathrm{r}^{2}=0.38, P=0.011\right.$ and $\mathrm{r}^{2}=0.29$, $P=0.031$, respectively), and percentage body fat $\left(\mathrm{r}^{2}=0.50\right.$,
$P=0.003)$. Biglycan gene expression was significantly elevated in visceral T2D compared to NGT $(P=0.002)$ and was significantly correlated with body weight $\left(\mathrm{r}^{2}=0.31\right.$, $P=0.024)$, plasma glucose concentration $\left(\mathrm{r}^{2}=0.46\right.$, $P=0.004)$, and percentage body fat $\left(\mathrm{r}^{2}=0.38, P=0.014\right)$ in visceral adipose tissue. This expression profile was similar to that of decorin.

Biglycan gene expression was measured in the same NGT, IGT, and T2D mesenteric and subscapular adipose tissue samples as decorin. This allowed us to analyze a potential association between the two genes during the development of obesity and T2D. Bivariate analysis revealed a strong correlation between decorin and biglycan gene expression $\left(\mathrm{r}^{2}=0.86\right.$, $P<0.001)$ during the development of obesity and T2D in P. obesus mesenteric and subscapular adipose tissue.

\section{Biglycan is predominantly expressed within stromal/vascular cells in fractionated adipose tissue}

To determine which cells in adipose tissue express biglycan, gene expression was measured in fractionated epididymal adipose

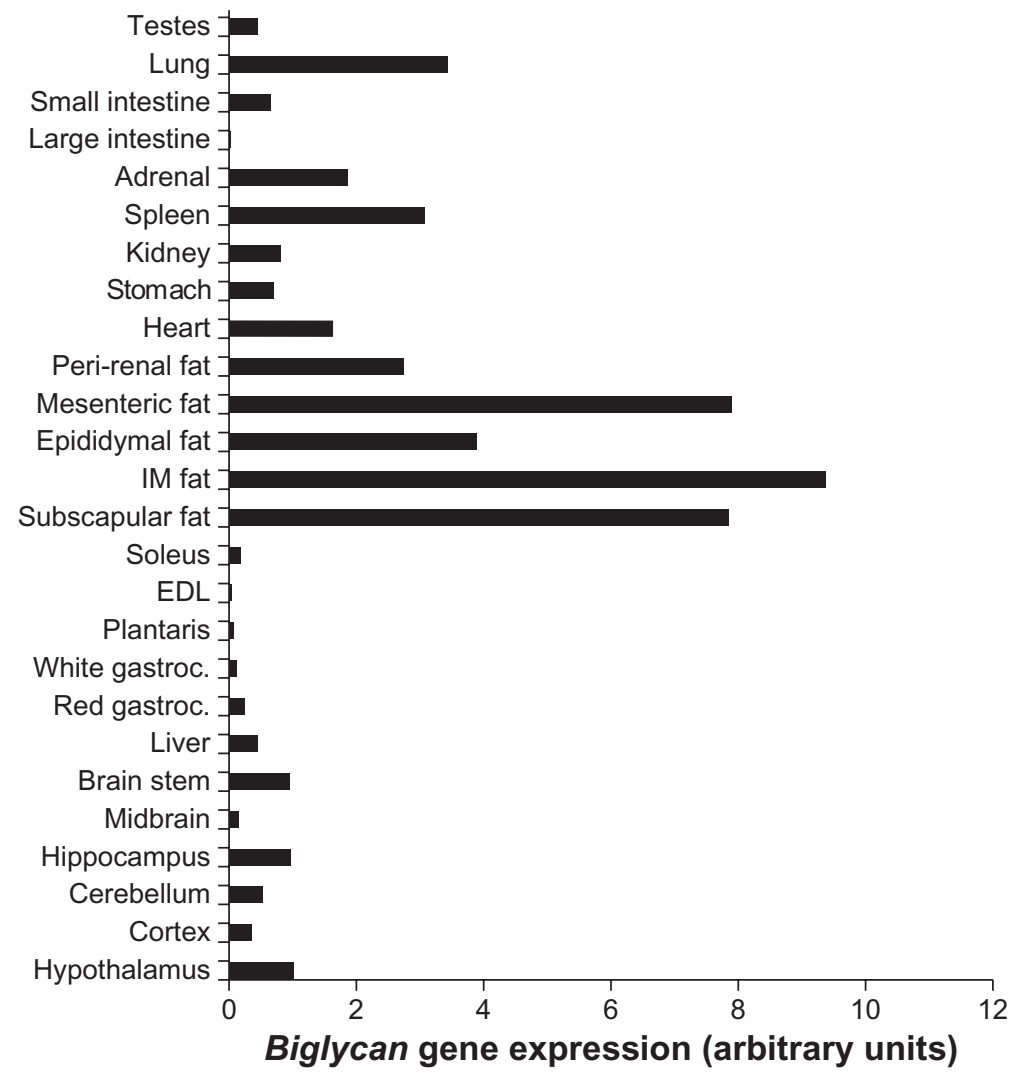

Figure I Biglycan gene expression in a variety of $P$. obesus tissues.

Notes: RNA was extracted from a variety of tissues from T2D P. obesus and gene expression measured by RT-PCR. Biglycan expression is represented relative to hypothalamic expression.

Abbreviations: P. obesus, Psammomys obesus; IM, intramuscular; EDL, extensor digitorum longus; gastroc, gastrocnemius; T2D, type 2 diabetes; RT-PCR, real time polymerase chain reaction. 


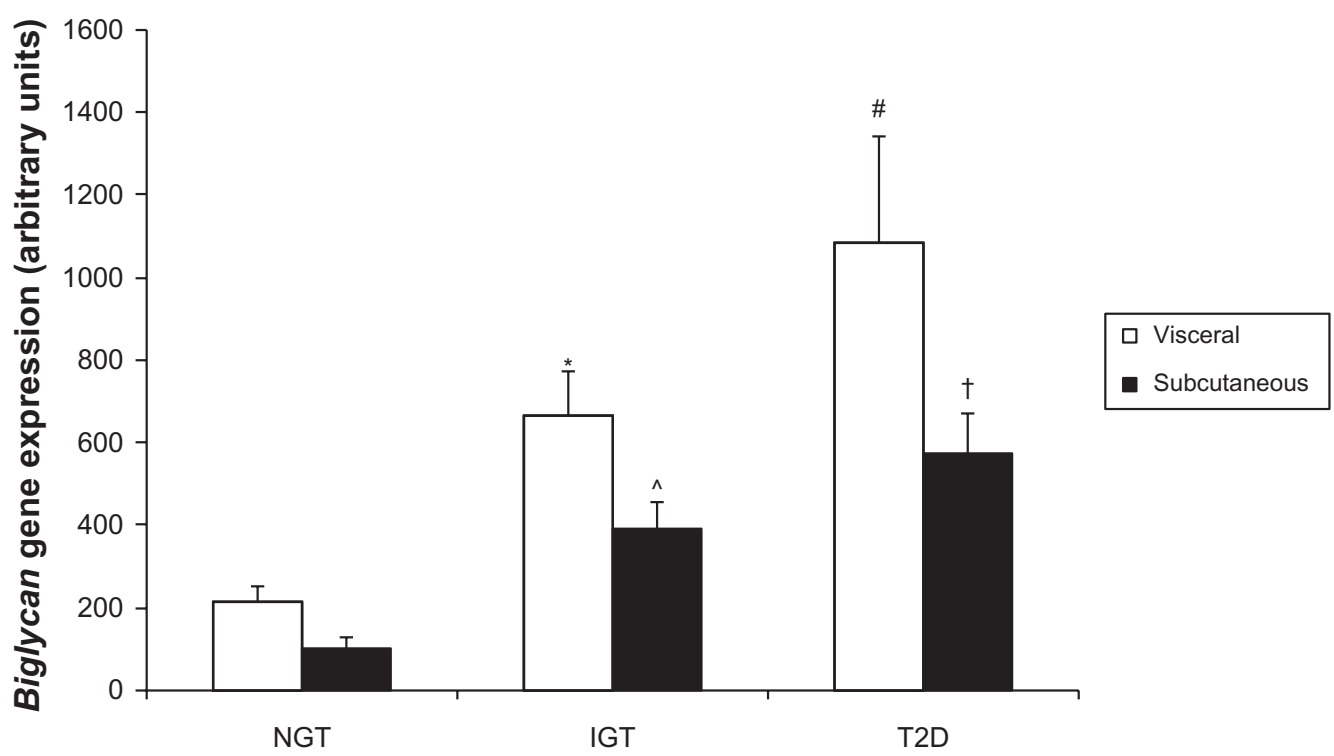

Figure 2A Analysis of biglycan gene expression in $P$. obesus visceral and subcutaneous adipose tissue.

Notes: RNA was extracted from visceral (mesenteric) and subcutaneous (subscapular) adipose tissue of NGT, IGT and T2D P. obesus and biglycan gene expression measured by RT-PCR. Data are mean \pm SEM ( $n=5-6$ per group). Data is represented relative to expression in NGT subcutaneous adipose tissue. $* P=0.049$ compared to subcutaneous IGT; $\wedge P=0.009,{ }^{\dagger} P<0.001$ compared to subcutaneous $N G T$, respectively; ${ }^{\sharp} P=0.002$ compared to visceral NGT.

Abbreviations: P. obesus, Psammomys obesus; NGT, normal glucose tolerant; IGT, impaired glucose tolerant; T2D, type 2 diabetes; RT-PCR, real time polymerase chain reaction; SEM, standard error of mean.

tissue from IGT P. obesus. There were two cellular populations - adipocytes and stromal/vascular cells. Comparable to decorin which had almost exclusive expression within stromal/vascular cells, biglycan was predominantly expressed in stromal/vascular cells compared to adipocytes $(P=0.023$, Figure 2B). Successful fractionation was confirmed using adipocyte-specific (leptin) and stromal/vascular-specific (CD68, Pref-1) markers. As expected, leptin gene expression was significantly higher in the adipocyte compared to the stromal/vascular fraction $(P<0.001)$, and CD68/Pref-1 gene expression was significantly higher in the stromal/vascular compared to adipocyte fraction $(P<0.001$; data not shown).

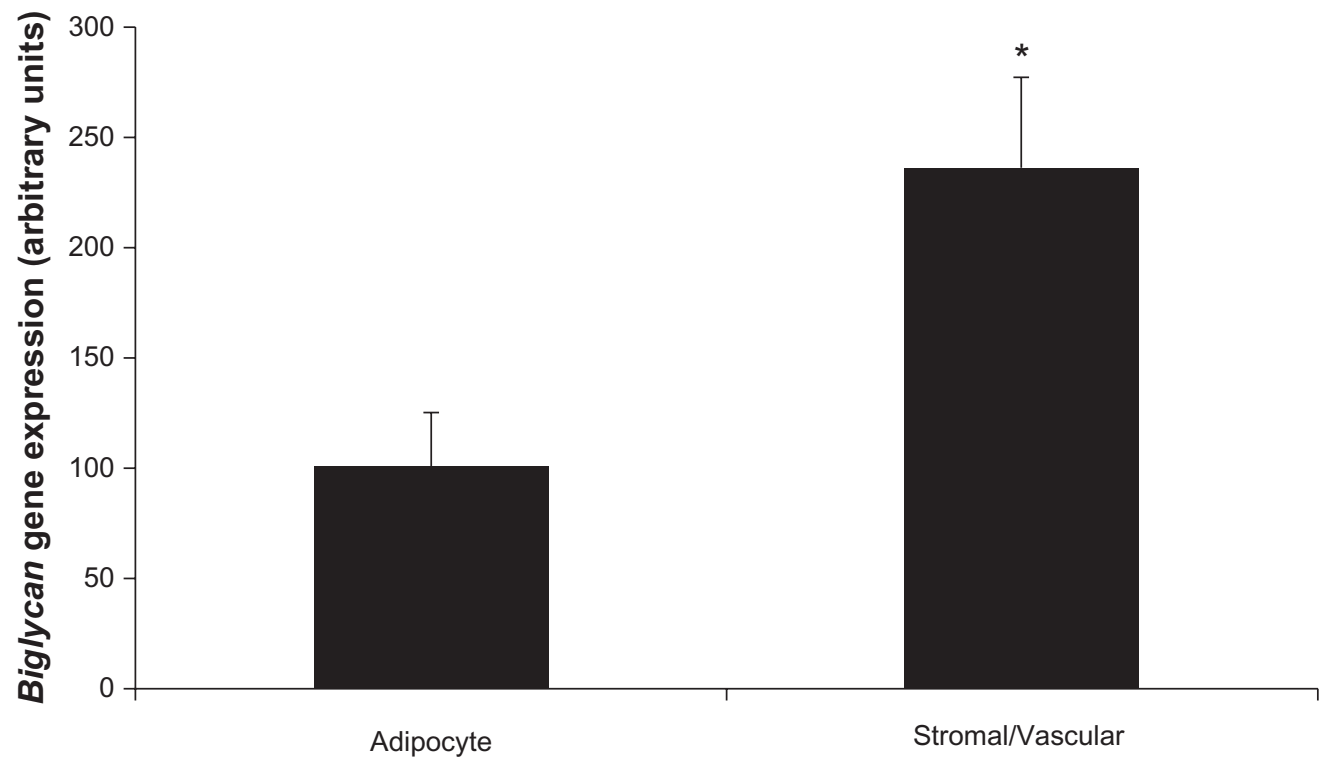

Figure 2B Biglycan expression in fractionated $P$. obesus epididymal adipose tissue.

Notes: Epididymal adipose tissue from $P$. obesus was fractionated into adipocytes and stromal/vascular cells and gene expression measured by RT-PCR. Data are mean \pm SEM ( $n=5$ per group). Overall, biglycan gene expression was significantly higher in the stromal/vascular compared to adipocyte fraction $(* P<0.023)$.

Abbreviations: P. obesus, Psammomys obesus; RT-PCR, real time polymerase chain reaction; SEM, standard error of mean. 


\section{Discussion}

We have previously shown the SLRP decorin to be highly expressed in adipose tissue, and upregulated in obesity suggesting a potential role in adipose tissue homeostasis or in the pathophysiology associated with obesity such as extracellular matrix (ECM) remodeling, inflammation, and angiogenesis within expanding adipose tissue. Here we extend these findings to include the closely related SLRP, biglycan, which also exhibits high expression in adipose tissue (and particularly visceral adipose tissue) compared to a wide variety of tissues examined, and increased expression in obesity.

Collectively, these data raise the possibility that increased expression of SLRPs in adipose tissue is a key feature during the development of obesity, T2D, and the metabolic syndrome. SLRPs are constituents of the ECM which undergoes constant degradation and renewal, ${ }^{15}$ allows the diffusion of nutrients, metabolites, and hormones between blood and cells within the tissue ${ }^{16}$ and functions not only as a scaffold for cells within tissues, but also as a reservoir of growth factors and cytokines and modulates their activities. ${ }^{17}$ SLRPs bind and interact with numerous proteins involved in matrix assembly, cell proliferation, and tissue morphogenesis. ${ }^{18}$ With regards to adipocyte differentiation, remarkable changes in cell morphology, cytoskeletal components, and the amount and type of ECM components secreted occurs. ${ }^{19}$ Furthermore, the development of obesity requires extensive reorganization of adipose tissue and the expression of genes involved in matrix remodeling was found to be increased in obese $\mathrm{db} / \mathrm{db}$ mice and resulted in the net accumulation of ECM components in parallel to increased adipocyte size. ${ }^{20}$ It has also been proposed that complications of diabetes are closely associated with changes in the $\mathrm{ECM}^{21,22}$ as macroangiopathic and microangiopathic complications resultant from the presence of diabetes have involved abnormalities within the extracellular intima. ${ }^{21}$

Given the above background information regarding SLRPs, it is therefore tempting to speculate that SLRPs may play a role in angiogenesis and ECM remodeling within expanding adipose tissue during the development of obesity and T2D. Recent studies have demonstrated biglycan to be upregulated in a murine model of diet-induced obesity with atherosclerosis, ${ }^{23}$ to be involved in vascular and proatherogenic remodeling in a rat model of metabolic syndrome, ${ }^{24}$ to be proangiogenic, ${ }^{25}$ and interestingly, to have upregulated expression in parallel with matrix degradation genes in white adipose tissue from $\mathrm{db} / \mathrm{db}$ mice fed a high fat diet. ${ }^{20}$ Biglycan has also been demonstrated to be upregulated in human omental compared to lean adipose tissue. ${ }^{26}$ This evidence builds upon the hypothesized role for biglycan in adipose tissue homeostasis, particularly in states of obesity where ECM remodeling is required for adipose tissue expansion.

Biglycan has been shown to be proinflammatory and acts through toll-like receptors in macrophages. ${ }^{27-29}$ Recent studies have supported a strong association of biglycan with inflammation, demonstrating increased levels of tumor necrosis factor $\alpha$ (a major inflammatory cytokine implicated in T2D) and biglycan in human omental adipose tissue, suggesting they may be coregulated. ${ }^{26}$ This potentially associates biglycan to obesity which is associated with a chronic low grade inflammatory state in adipose tissue (particularly visceral adipose tissue), ${ }^{30,31}$ and the subsequent development of insulin resistance and T2D. ${ }^{30}$ However, these are postulated theories, therefore additional experiments to explore a role in inflammation and substantiate these theories are now required.

In summary, our data suggest that the family of SLRPs may play a role in the development of obesity and T2D, with a hypothesized role of facilitating the expansion of adipose tissue mass within an animal model. Further work is now required to corroborate these results within humans.

\section{Acknowledgments}

This work was funded by Verva Pharmaceuticals Ltd. This manuscript contains original research and has not been previously published in another journal.

\section{Disclosure}

The authors of this manuscript have no conflicts of interest to disclose and have approved the final article submitted to Biologics: Target and Therapeutics. Author contribution as follows: KB project design, data collection and analysis, preparation of manuscript; DS/KW: project design and editorial assistance with manuscript. All authors have read and approved this manuscript.

\section{References}

1. Bolton K, Segal D, McMillan J, et al. Decorin is a secreted protein associated with obesity and type 2 diabetes. Int J Obes (Lond). 2008;32(7): 1113-1121.

2. Fisher LW, Termine JD, Young MF. Deduced protein sequence of bone small proteoglycan I (biglycan) shows homology with proteoglycan II (decorin) and several nonconnective tissue proteins in a variety of species J Biol Chem. 1989;264(8):4571-4576.

3. Hildebrand A, Romaris M, Rasmussen LM, et al. Interaction of the small interstitial proteoglycans biglycan, decorin and fibromodulin with transforming growth factor beta. Biochem J. 1994;302(Pt 2):527-534.

4. Schonherr E, Witsch-Prehm P, Harrach B, Robenek H, Rauterberg J, Kresse H. Interaction of biglycan with type I collagen. J Biol Chem. 1995;270(6):2776-2783.

5. Bi Y, Stuelten $\mathrm{CH}$, Kilts T, et al. Extracellular matrix proteoglycans control the fate of bone marrow stromal cells. J Biol Chem. 2005;280(34): 30481-30489. 
6. Young MF, Bi Y, Ameye L, Chen XD. Biglycan knockout mice: new models for musculoskeletal diseases. Glycoconj J. 2002;19(4-5): 257-262.

7. Wadhwa S, Bi Y, Ortiz AT, et al. Impaired posterior frontal sutural fusion in the biglycan/decorin double deficient mice. Bone. 2007;40(4): 861-866.

8. Danielson KG, Baribault H, Holmes DF, Graham H, Kadler KE, Iozzo RV. Targeted disruption of decorin leads to abnormal collagen fibril morphology and skin fragility. J Cell Biol. 1997;136(3):729-743.

9. $\mathrm{Xu} \mathrm{T,} \mathrm{Bianco} \mathrm{P,} \mathrm{Fisher} \mathrm{LW,} \mathrm{et} \mathrm{al.} \mathrm{Targeted} \mathrm{disruption} \mathrm{of} \mathrm{the} \mathrm{biglycan}$ gene leads to an osteoporosis-like phenotype in mice. Nat Genet. 1998;20(1):78-82.

10. Corsi A, Xu T, Chen XD, et al. Phenotypic effects of biglycan deficiency are linked to collagen fibril abnormalities, are synergized by decorin deficiency, and mimic Ehlers-Danlos-like changes in bone and other connective tissues. J Bone Miner Res. 2002;17(7):1180-1189.

11. Ameye L, Young MF. Mice deficient in small leucine-rich proteoglycans: novel in vivo models for osteoporosis, osteoarthritis, Ehlers-Danlos syndrome, muscular dystrophy, and corneal diseases. Glycobiology. 2002;12(9):107R-116R.

12. Chen XD, Fisher LW, Robey PG, Young MF. The small leucinerich proteoglycan biglycan modulates BMP-4-induced osteoblast differentiation. Faseb J. 2004;18(9):948-958.

13. Casar JC, McKechnie BA, Fallon JR, Young MF, Brandan E. Transient up-regulation of biglycan during skeletal muscle regeneration: delayed fiber growth along with decorin increase in biglycan-deficient mice. Dev Biol. 2004;268(2):358-371.

14. Barnett M, Collier GR, Collier FM, Zimmet P, O'Dea K. A crosssectional and short-term longitudinal characterisation of NIDDM in Psammomys obesus. Diabetologia. 1994;37(7):671-676.

15. Rahmani M, Wong BW, Ang L, et al. Versican: signaling to transcriptional control pathways. Can J Physiol Pharmacol. 2006;84(1): 77-92.

16. Alberts B, Bray D, Lewis J, Raff M, Roberts K, Watson JD. Molecular Biology of the Cell. 3rd ed: Garland Publishing; 1994.

17. Kresse H, Schonherr E. Proteoglycans of the extracellular matrix and growth control. J Cell Physiol. 2001;189(3):266-274.

18. Iozzo RV, Murdoch AD. Proteoglycans of the extracellular environment: clues from the gene and protein side offer novel perspectives in molecular diversity and function. Faseb J. 1996;10(5):598-614.
19. Smas CM, Sul HS. Control of adipocyte differentiation. Biochem J. 1995;309(Pt 3):697-710.

20. Huber J, Loffler M, Bilban M, et al. Prevention of high-fat diet-induced adipose tissue remodeling in obese diabetic mice by $n-3$ polyunsaturated fatty acids. Int J Obes (Lond). 2007;31(6):1004-1013.

21. Olsson U, Bondjers G, Camejo G. Fatty acids modulate the composition of extracellular matrix in cultured human arterial smooth muscle cells by altering the expression of genes for proteoglycan core proteins. Diabetes. 1999;48(3):616-622.

22. Lehti TM, Silvennoinen M, Kivela R, Kainulainen H, Komulainen J. Effects of streptozotocin-induced diabetes and physical training on gene expression of extracellular matrix proteins in mouse skeletal muscle. Am J Physiol Endocrinol Metab. 2006;290(5):E900-E907.

23. King VL, Hatch NW, Chan HW, de Beer MC, de Beer FC, Tannock LR. A murine model of obesity with accelerated atherosclerosis. Obesity (Silver Spring). 2010;18(1):35-41.

24. Mangat R, Su J, Scott PG, Russell JC, Vine DF, Proctor SD. Chylomicron and apoB48 metabolism in the JCR:LA corpulent rat, a model for the metabolic syndrome. Biochem Soc Trans. 2007;35(Pt 3): $477-481$.

25. Zhang W, Chuang YJ, Jin T, et al. Antiangiogenic antithrombin induces global changes in the gene expression profile of endothelial cells. Cancer Res. 2006;66(10):5047-5055.

26. Ward M, Ajuwon KM. Regulation of pre-adipocyte proliferation and apoptosis by the small leucine-rich proteoglycans, biglycan and decorin. Cell Prolif. 2011;44(4):343-351.

27. Moreth K, Brodbeck R, Babelova A, et al. The proteoglycan biglycan regulates expression of the $\mathrm{B}$ cell chemoattractant CXCL13 and aggravates murine lupus nephritis. J Clin Invest. 2010;120(12):4251-4272.

28. Babelova A, Moreth K, Tsalastra-Greul W, et al. Biglycan, a danger signal that activates the NLRP3 inflammasome via toll-like and P2X receptors. J Biol Chem. 2009;284(36):24035-24048.

29. Schaefer L, Babelova A, Kiss E, et al. The matrix component biglycan is proinflammatory and signals through Toll-like receptors 4 and 2 in macrophages. J Clin Invest. 2005;115(8):2223-2233.

30. Torres-Leal FL, Fonseca-Alaniz MH, Rogero MM, Tirapegui J. The role of inflamed adipose tissue in the insulin resistance. Cell Biochem Funct. 2010;28(8):623-631.

31. Wellen KE, Hotamisligil GS. Inflammation, stress, and diabetes. J Clin Invest. 2005;115(5):1111-1119.
Biologics: Targets \& Therapy

\section{Publish your work in this journal}

Biologics: Targets \& Therapy is an international, peer-reviewed journal focusing on the patho-physiological rationale for and clinical application of Biologic agents in the management of autoimmune diseases, cancers or other pathologies where a molecular target can be identified. This journal is indexed on PubMed Central, CAS, EMBase, Scopus

\section{Dovepress}

and the Elsevier Bibliographic databases. The manuscript management system is completely online and includes a very quick and fair peerreview system, which is all easy to use. Visit http://www.dovepress. com/testimonials.php to read real quotes from published authors. 